\title{
Brown Dwarfs in the UKIRT Infrared Deep Sky Survey (UKIDSS)
}

\author{
Nigel Hambly ${ }^{1}$ \\ Wide Field Astronomy Unit, Institute for Astronomy, University of \\ Edinburgh, Royal Observatory, Blackford Hill, Edinburgh EH9 $3 \mathrm{HJ}$
}

\begin{abstract}
During the final quarter of 2003, UKIRT will take delivery of WFCAM. This new wide-field camera will have a FOV of 0.2 square degrees, and is therefore well suited to large-scale survey work. A consortium of more than 60 astronomers has successfully bid for a large fraction of all UKIRT time over the next 5 years to undertake several public surveys using this new facility. These surveys are collectively known as the UKIRT Infrared Deep Sky Survey (UKIDSS). In this short paper I will describe the project and review the prospects for BD research using UKIDSS data, highlighting some of the major science goals related to BDs that we hope will be achieved.
\end{abstract}

\section{Introduction}

A new wide-field camera (WFCAM) for the $3.8 \mathrm{~m}$ United Kingdom Infrared Telescope (UKIRT) is being designed and built at the UK ATC in Edinburgh (technical details can be found at http://www.roe.ac.uk/projects/wfcam). The camera uses $42048 \times 2048$ Rockwell Hawaii-2 detectors, $90 \%$ spaced in the focal plane, with a pixel scale of 0.4 arcsec pix ${ }^{-1}$ to yield an instantaneous fieldof-view of 0.2 sq. deg. For point sources in median seeing, the $5 \sigma$ sensitivities in a 1 hour integration are calculated as $\mathrm{J}=22.1, \mathrm{H}=21.2$ and $\mathrm{K}=20.8$ (this allows for a $2 \times 2$ 'microstep' sequence at any given position to adequately sample the PSF at the focal plane; all magnitudes are quoted in the Vega system).

The typical data-rate of WFCAM will be $\sim 100$ Gbyte per night with peak data rates being more than twice that figure. Over the lifetime of the instrument, 100s of Tbytes of data will be accumulated. In order to process and calibrate this huge volume of data automatic pipeline processing is being designed and implemented at the Cambridge Astronomical Survey Unit (see http://www.ast.cam.ac.uk/ ${ }^{\sim m i k e / c a s u) . ~ F u r t h e r m o r e, ~ i n ~ o r d e r ~ t o ~ e n-~}$ able the fullest possible scientific exploitation of survey data generated by the instrument, a Science Archive (Lawrence et al. 2003) is being developed and implemented for WFCAM at the Wide Field Astronomy Unit in Edinburgh (see also http://www.roe.ac.uk/wfau).

${ }^{1}$ On behalf of the UKIDSS consortium 
In recognition of the enormous potential that WFCAM has for wide-angle surveys, and in view of the great success of the legacy Schmidt photographic surveys of the past half-century, an international consortium of more than 60 astronomers has assembled and developed an ambitious survey programme for WFCAM. This proposal is now known as the UKIRT Infrared Deep Sky Survey (UKIDSS), e.g. Warren (2002); see also http://www.ukidss.org/. The combined science case of UKIDSS details a programme that is currently unrivalled in terms of depth, field-of-view and therefore survey volume. UKIDSS proposes a nested series of surveys ranging from the Large Area Survey ('LAS', 4000 sq. deg. to $K=18.4$ ), the Galactic Plane Survey ('GPS', 1800 sq. deg. to $K=19$ ), the Galactic Clusters Survey ('GCS', 1600 sq. deg. to $K=18.7$ ), the Deep Extragalactic Survey ('DXS', 35 sq. deg. to $K=21$ ) to the Ultra-Deep Survey ('UDS', 0.8 sq. deg. to $\mathrm{K}=23$ ). Amongst the diverse science goals are i) finding the nearest and faintest BDs; ii) determining the form of the substellar IMF; and iii) finding Population II BDs. Membership of the UKIDSS consortium is open, but confers no special proprietary rights to the survey data. Within ESO, the data will be freely available to any astronomers in all member states (including the UK). After an appropriate proprietary period (yet to be agreed) the survey data will be freely available worldwide.

\section{Prospects for BD research in UKIDSS}

The LAS component of UKIDSS is designed to cover $40 \%$ of the SDSS-surveyed area (York et al. 2000) to depths some 4 to 6 magnitudes deeper than 2MASS and DENIS. We expect to discover examples of the smallest/coolest BDs as well as possibly the nearest BD. For example, an $m \sim 5(10) \mathrm{M}_{\mathrm{JUP}} \mathrm{BD}$ will be detectable at $\sim 2$ pc if aged 0.5(1.0) Gyr. Tens of thousands of $L$ dwarfs will be identified. Hundreds of T-dwarfs will also be found by employing cuts in multicolour space; colours (or colour limits) from combinations of i'z'YJHK (where $\mathrm{Y}$ is a new $\sim 1 \mu \mathrm{m}$ filter specified within UKIDSS to distinguish the highest redshift QSOs from the coolest BDs) will be available for these purposes. Two passes at J band, separated by $\sim 2 \mathrm{yr}$, will be made within the LAS to provide proper motion information. In this way, we expect to be able to identify a handful of higher mass, high velocity Population II BDs.

The GPS will survey the Galactic Plane accessible to UKIRT to latitudes $-5^{\circ}<b<+5^{\circ}$. Again, multi-epoch imaging will enable identification of cool, nearby sources from proper motions. Specific questions to be addressed by the GPS data include investigation of any young, low scale-height, higher space density population of BDs, as well as any serendipitous discovery of very cool, very nearby objects.

The GCS is the UKIDSS component specifically aimed at BD science. Figure 1 presents the scientific justification in a nutshell: the questions to be answered are the form of the substellar IMF, and its universality (or otherwise dependence on environment) around the local regions of the Galaxy. To do this, we will survey 10 of the nearest young stellar clusters and star formation regions where we expect to discover tens to hundreds of member BDs (depending on the target and the form of the IMF). The GCS targets are: Alpha-Per, the Pleiades, the Hyades, Praesepe, Coma-Ber and IC4665 (open clusters) along 


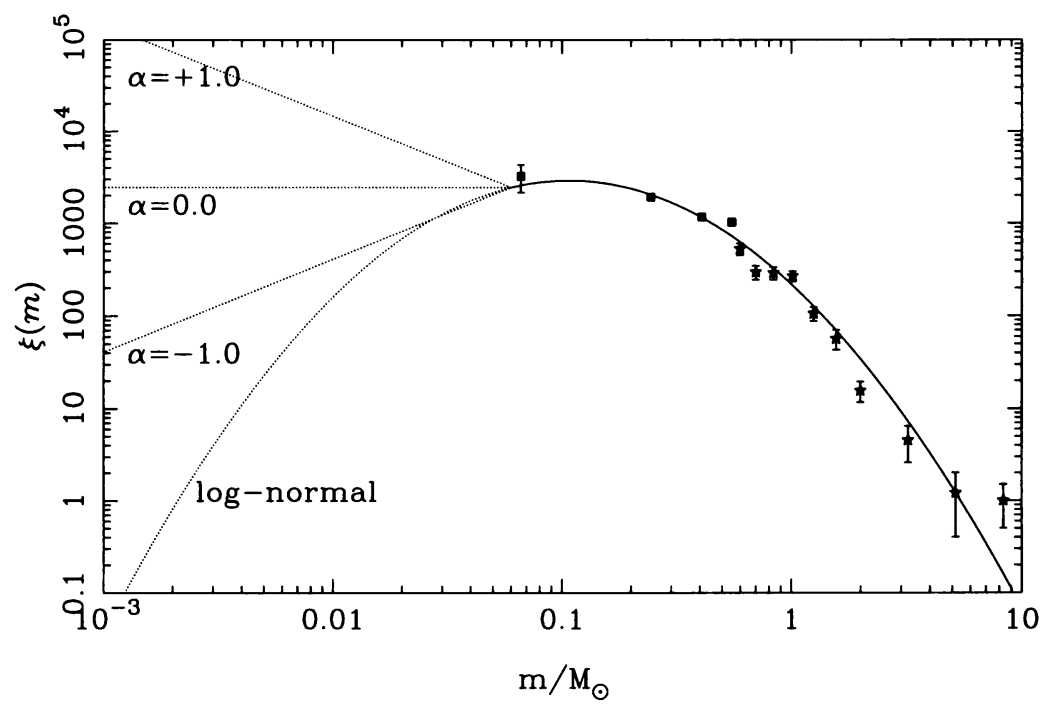

Figure 1. Current uncertainties in the form of the substellar IMF. There is as yet no consensus on the form (power-law, log-normal) of the substellar IMF; it is presently unclear whether we are seeing evidence for a variation in the IMF between different star formation sites. The UKIDSS GCS aims to address this fundamental problem by surveying 10 clusters and star formation regions in a uniform way. The data points above are from the Pleiades (see Hambly et al. 1999) along with a log-normal fit (solid line); dotted lines indicate various extrapolations and the current large uncertainties

with Perseus-OB2, Taurus-Auriga, Orion and Upper Sco in Scorpius-OB2. In this way we will obtain adequate samples to define the IMF in each case - there is still no consensus as to the form of the IMF and its universality (e.g. articles elsewhere in these proceedings).

Finally, the DXS and UDS components also have the potential for contributing to BD science, although their primary scientific goals are of course extragalactic. Deep pencil-beam surveys are certainly not optimal for volume samples of rare objects but do have the potential for making serendipitous discoveries. The DXS and UDS will also yield information on the scale height of the disk L-dwarf population. For example, in Figure 2 we show the results of computations from Chabrier (2002) for a UDS-like survey to $J=25$ over 1 sq. deg. for two reasonable IMFs. These 'discovery functions' indicate the number of BDs that are expected per sq. deg. per unit $\mathrm{J}$ magnitude at the Galactic latitude of the Subaru Deep Field $\left(b=-60^{\circ}\right)$. While such data may not distinguish clearly between different disk population IMFs, they will certainly yield samples adequate for scale height studies.

Acknowledgments. I thank Gilles Chabrier for making the calculations for the results presented in Figure 2. I acknowledge the combined efforts of the WFCAM project team at the UK ATC, the UKIDSS consortium, and my col- 


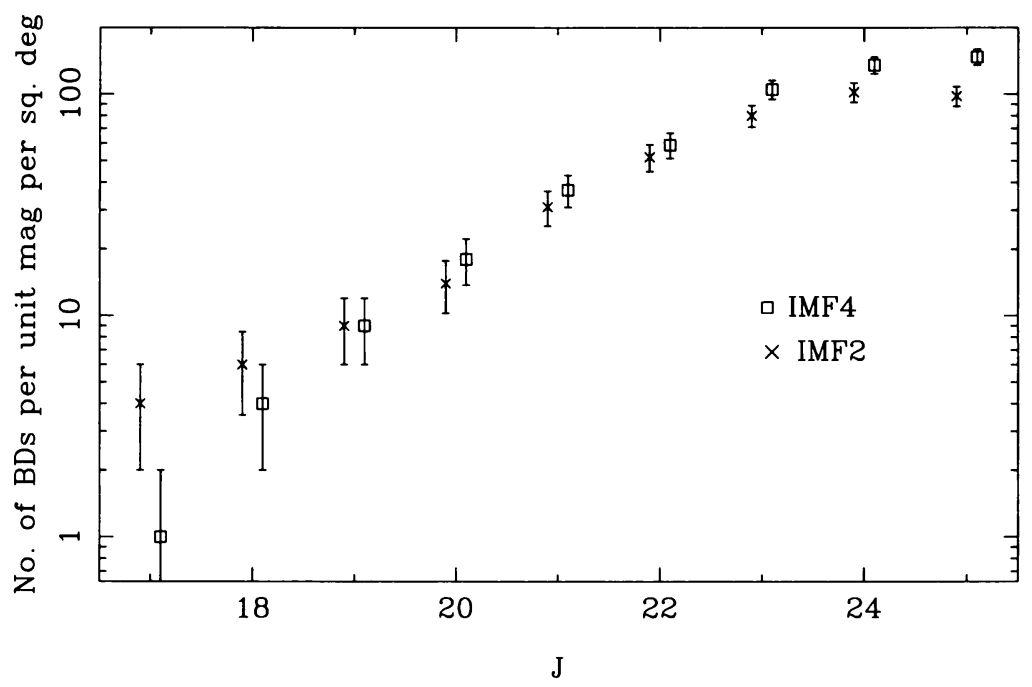

Figure 2. Model predictions of the number of BDs that will present in the UDS (after Chabrier 2002).

leagues at CASU and WFAU, without whom the new infrared surveys described here would be impossible.

\section{References}

Chabrier, G. 2002, ApJ, 567, 304

Hambly, N. C., Hodgkin, S. T., Cossburn, M. R., Jameson, R. F. 1999, MNRAS, 303,835

Lawrence, A., Hambly, N. C., Mann, R. G., Irwin, M. J., Lewis, J. R., McMahon, R. G., Adamson, A. 2003, Proc. SPIE vol. 4836, in press

Warren, S. J. 2002, ESO Messenger, No. 108, 31

York, D. G. et al. 2000, AJ, 120, 1579 